\title{
Assessment of Knowledge about the BARC Centre and Satisfaction with the Educational Services Available.
}

\author{
Paul Bacon ${ }^{1 *}$ and Shkumbim A Thaqi ${ }^{2}$ \\ ${ }^{1}$ Brimingham Research Arthritis Center, UK \\ ${ }^{2}$ Center for Family Medicine, Kosovo
}

Submission: September 08, 2016; Published: September 28, 2016

*Corresponding author: Paul Bacon, Brimingham Research Arthritis Center, UK.

\begin{abstract}
The aim if this proposal is to determine knowledge in the general population about arthritis and immunology disorders ; to ascertain if this has improved in the decade since our Needs Assessment; and the satisfaction with the educational material now available to support communities and arthritis sufferers.
\end{abstract}

\section{Background}

BARC (Birmingham Arthritis Resource Centre) was set up to provide education and support to people with arthritis and their carers, based on a formal Needs Assessment. BARC aims to promote self-coping to help people to deal with the physical and social disabilities caused by their disease. It is cited in the city centre public library and works alongside the regular medical NHS service provision. Services for Rheumatology have always had lower priority and funding than those for acute services such as Cancer and Heart disease. In addition they have historically been somewhat restricted in the West Midlands (the UK region where Birmingham is the central city) compared to the rest of the UK. The picture is also complicated by the high percentage of ethnic minority groups locally (generally referred to as BME groups- Black and Minority Ethnic). Birmingham is set to become the first major UK city where BME groups will become the majority within the next ten years. There is evidence that "excluded groups" - such as immigrants, the poor and the less-well educated have poorer health but do not access the NHS in the same way as the white middle-class population for a variety of reasons, including cultural, language and poverty barriers. This is clearly relevant to the wider European scene where there is increasing pressure from immigration while currently both health and social programs are threatened by the financial recession.

The BARC project was started a decade ago with a formal research process to determine the extent of current services and what people wanted. This "Needs Assessment" showed that both medical profession and public perceived a need for more information provided in an informal setting (ie a non-medical setting) - and wanted it in a range of languages Adab et al. [1].
There was also a widespread desire for more support services for patients. The BARC centre was set up on the basis of this in space provided by the City in the Central Library and is manned by volunteers. These have been selected and trained by the Centre manager, Chan Gordhan, who has a long background in social and voluntary work. The volunteers come from a range of ethnic backgrounds and importantly they have all had some personal rheumatic problem. Thus they fit what the UK government is now calling "expert patients" - and promoting the idea that they are best placed to help others since they have learnt how to cope. Interestingly our experience shows that volunteering to help others also empowers them to deal with their own lives, so they should also be the best placed group to teach us how to empower our clients. Our data also shows that the BARC service is wanted as well as needed locally.

The key point in developing any new service is to provide an evidence base for it. BARC set out to do this from the outset. Following the initial "Needs Assessment" We carried out a focus group study to determine what patients from BME groups were looking for from the local health services Bacon et al. [2]. A key factor expressed by the participants was the desire to be listened too. They were dissatisfied with their doctors who were seen to lack time to take in the patients broader complaints. This echoes wider concerns about poor doctor patient communications an area which the Royal College of Physicians is holding an enquiry into at present.

BARC has set up sympathetic listening as one of the basic parts of the service Gordhan $[3,4]$. This is provided by trained volunteers. They are themselves patients and come from a range of ethnic and linguistic backgrounds, so that they are able to 
provide culturally sensitive guidance to clients. We have collected data on who has attended and how satisfied they are with the service provided Treharne [5]. Approximately 40\% of attendees come from the BME groups, similar to the general population. Thus we are getting through to target populations - but not in large enough numbers. We have also had high gradings for client satisfaction.

We have also addressed the need for relevant patienteducation material understandable to those for whom English is not their mother tongue. We recently completed a set of educational leaflets, designed as "bottom-up" material that is based on questions people actually ask rather than information doctors think patients ought to know. They are in simple English, avoiding technical terms, so as to be easily understood. The first six have been translated into Urdu and recorded on CD's in both languages, as well as in print format with a few cartoons to illustrate them. A preliminary piece of market research in the BARC Centre shows that the volunteers think they are what is needed and a small sample of clients listening to the first one agreed. The Urdu translation has also been approved by a range of Indian colleagues as being both true to the English information and understandable by a range of local language speakers. The translation is not strict Urdu but includes phrases used in Bollywood films (watched by all the local S. Asian groups) as well as some English words generally used in the version of "Urdu" widely used around Birmingham.

A questionnaire-based assessment of the first of these CD's - on Understanding Arthritis - showed that clients gave it high scores for clarity of information and obtaining information that they wanted. In general they found the CD helped them to cope Sharif [6]. We are just completing an assessment of the CD on rheumatoid arthritis and the outcome is very exciting. The challenge was far greater here as the usefulness of the CD was examined in a specialist RA clinic which already had a highly trained specialist nurse providing explanations and support to patients. Despite this the comments made at the focus groups demonstrated that the study participants had found the additional BARC service a major help Kumar et al. [7]. There is now patient pressure to set up such a service on a regular basis in the hospital setting. This would be in line with the recent Report from the influential Kings Fund which noted a lack of understanding on the quality of RA care and the struggle many RA patients have to access quality care Kings Fund [8].

In the same way, we have struggled to reach our target for new attendees at the BARC centre, despite the evidence for the need for and the success of the BARC service. Total numbers accessing the BARC service, including phone calls and web-site hits, have increased year-on year but surprisingly there has been no increase in personal visitors. A number of community centres have asked for the manager to go out to specific groups with promotional and educational talks. This alternative approach has proved very popular but many attendees have said they were not aware of the BARC centre. These outreach sessions are demanding on Chan Gordhan's time and there is an excellent service available at the library. Thus the next essential step is a study of why people are not coming in the predicted numbers.

\section{Hypothesis}

We propose that the population in general tend to downplay the importance of their musculo-skeletal problems. This is reinforced by the poor publicity that arthritic diseases get compared to some others. Analysis of the relative importance given by press or TV showed that heart disease and cancer got far more attention and were treated as serious scientific problems. Rheumatic diseases by contrast were seen as "lifestyle problems" for which there was no real medical treatment. The existence of a ground-breaking local service does not appear to have changed that mould to any major extent. Each time that the BARC Centre has been discussed on local radio there has been a sharp rise in client enquiries - but only for a short period. We intend to analyse the degree of local awareness of the BARC and at the same time look further into the responses of those who do actually come to seek help.

\section{Methods}

The first aspect will be carried out by collecting data about knowledge of BARC and satisfaction with current educational support using standardised questionnaires. This will target both a random population (people accessing the Central Library for any purpose) and specific communities such as local Sikh and Somali populations who have already identified a perceived need for an increased service for their groups. A minimum of 200 library people will be sampled at random in each grouping. The second part (analysis of satisfaction with current services) will be completed by analysis of the data collected over the past two years from attendees at the Centre, who are all asked to complete such a form. The data from this project will be compared to that obtained 10 years ago in the original Needs Assessment

\section{Broader aspects of Fellowship}

The advantages of taking on this project would be to widen your experience into qualitative research and introduce you to a new but important area of rheumatology, patient education. The latter has many messages for someone practicing in a major city with an immigrant population and you have already reported working with several ethnic minority groups in Kosova. We have been thinking about this project for some time, so there are some things already in place to facilitate your research. We have already trailed a simple questionnaire for these assessments. A sociology student is currently using these to collect some preliminary data from library visitors. That experience will focus the further development of the project. A trained health psychologist is available to help with analysing the questionnaires and the unstructured material coming from 
the "free comment" section at the end of each form. In the same way, the set of forms collected from clients attending the Centre in its early years have been analysed and will form a useful comparison with the planned analysis of the comments collected from recent clients.

This exercise will definitely lead to at least one published paper. The methodologies used will be of value to you in assessing the worth of conventional treatment options across the field of rheumatology. Our speciality deals with incurable chronic disease and there is increasing evidence that patients have a different perspective on the outcome to their doctors Hewlett [9]. Helping people to cope with chronic disability, improving their life by addressing their real concerns rather than measuring "medical outcomes" like degree of swelling or ESR, is becoming increasingly important. Finding ways of reaching out to the large percentage of the population who have a disability related to a rheumatic problem is also essential to persuade politicians to take the subject seriously and invest in it. Thus the experience gained from this would be advantageous to your career in many ways - and I believe you would find working in BARC both interesting and rewarding. Once in place here you can join in all the usual University Rheumatology Departmental activities, from seminars to clinical meetings. We would also work to get you some exposure to Rheumatoid Arthritis clinics as an observer on an informal basis. That will be easier to do with colleagues on the ground than to set up formally in advance with the current NHS bureaucracy [10].

\section{Conclusion}

You will have free time to catch up on your reading, particularly on the fairly large literature on self-coping and on what people expect from health services. You would need this to write a good paper and I would expect you to write up a comprehensive introduction and methods section well before data collection has been completed. Of course we will be available to discuss that with you but it will be your responsibility to produce the first version. I believe that an important part of such a fellowship is learning how to plan and write up your own research projects for the future.

\section{References}

1. Adab P, Rankin EC, Witney AG, Miles KA, Bowman S, et al. (2004) Use of a corporate needs assessment to define the information requirements of an arthritis resource centre in Birmingham: comparison of patients and professional views. Rheumatology (Oxford) 43(12): 1513-1518.

2. Paul A Bacon, Chandrika Gordhan, Elizabeth D Hale, Gareth J Treharne, George D Kitas (2005) Extending Choice for patients with Arthritis. Report prepared for Strategic Health Authority by Birmingham Arthritis Resource Centre.

3. Chandrika Gordhan, Roz Farr, Paul Bacon (2008) Its not just about diagnosis-learning to live with chronic disease. Rheumatology (Oxford) 47(2): 506.

4. Gordhan C (2003) The Birmingham Arthritis Resource Centre: meeting multicultural information needs. British Journal of Therapy and Rehabilitation 10(9): 394.

5. Treharne GJ, Batt B, Hale ED, Witney AG, Gordhan C, et al. (2004) Assessing patient, carer and healthcare professional education provided by the Birmingham Arthritis Resource Centre (BARC). Rheumatology (Oxford) 43(12): 1594-1595.

6. Fazeelat Sharif, Chandrika Gordhan, Elizabeth Hale, Paul Bacon (2008) Assessment of multi-lingual information CD's produced by Birmingham Arthritis Resource Centre. Rheumatology (Oxford) 47(S2): 117.

7. Kanta Kumar, Karim Raza, Chandrika Gordhan, Paul Bacon (2009) Educational support service plus audio CD breaks barriers for South Asian patients with RA: an assessment of the BARC model.

8. Perceptions of patients and professionals on rheumatoid arthritis care. King's Fund Report Jan2009.

9. Sarah Hewlett (2003) Patients and Clinicians have Different Perspectives on Outcomes in Arthritis. J Rheumatology 30: 877-879.

10. Richard McComb (2008) Centre where life begins after diagnosis-Buzz feature-University of Birmingham paper 102: 6 . 